\title{
Estudio Evaluativo de los Metales Contaminantes y sus Alternativas de Prevención
}

\section{Evaluative Study of Metal Contaminants and Preventive Alternatives}

\author{
1 Julio Vargas Paniagua
}

\begin{abstract}
RESUMEN:
El desarrollo del presente trabajo se enfoca principalmente en el estudio de la contaminación que sufren las personas y el medio ambiente, por la presencia de metales pesados. Los objetivos fueron: Evaluar y e identificar los agentes contaminantes que provocan diferentes enfermedades profesionales. Para el desarrollo de la evaluación y su prevención clinico-médico, se han estudiado en particular cinco metales pesados: Plomo, Mercurio, Arsénico, Cadmio y Asbesto, que al estar expuestos generan patologías diferentes y muy severas que hasta pueden causar la muerte. Por ultimo se analiza la salubridad de las personas que estén expuestas a estos metales que en la industria minera se trabajan ya sea como materia prima o producto final, analizando sus patologias que desencadenan según la concentración de los contaminantes y proponiendo tratamiento médico, con el propósito de ilustrar alternativas de prevención.
\end{abstract}

Palabras clave: metales pesados, patologia, salubridad, contaminación.

\begin{abstract}
:
The development of this research focuses primarily on the study of pollution suffered by people and the environment, due to the presence of heavy metals. The objectives were: to assess and identify the pollutants that cause different diseases. In order for the evaluation to develop as well as its clinical prevention, a group of five heavy metals has been studied in particular: lead, mercury, arsenic, cadmium and asbestos, all of which being exposed generate a number of severe pathologies that might even cause death. Finally there is a discussion about the health of those persons who are exposed to these metals working in mining industry either as raw material or finished product, analyzing the pathologies produced in certain concentrations of pollutants and proposing medical treatment with the purpose of illustrating alternatives for prevention.
\end{abstract}

Keywords: heavy metals, pathology, health, pollution. 


\section{INTRODUCCIÓN}

Los metales pesados que se obtienen en la minería peruana por lo general generan un ambiente de contaminación. Existen soluciones para estos problemas porque hoy se vienen implementando tecnologías de punta, que permiten minimizar el riesgo ambiental minero. Existen legislaciones, y sobre todo una genuina voluntad por parte de las grandes compañías mineras internacionales de "hacer las cosas bien", es decir por una minería responsable. La contaminación se da no solo en la industria minera, sin embargo toma gran importancia porque de por medio interviene el aspecto social y se juegan muchos interés y hoy mas que nunca, por los precios que se encuentran muy altos en el mercado.

\section{Objetivos}

Realizar un estudio de los principales metales pesados que se producen en la industria minera.

Evaluar la contaminación que dañen la salud de las personas

Evaluar su patología de las personas expuestas y tratamiento alternativo

\section{MATERIALES Y MÉTODOS}

\subsection{Metodología}

Se utilizó la metodología de investigación globalizada de campo, apoyándose a fuente bibliográfica, apoyo científico a nivel universal, etc. realizando las consultas en base a experiencias avanzadas llegando a conclusiones de un nivel que justifique el presente trabajo de investigación.

Además la metodología está basado a la investigación de diferentes casos que han producido inclusive la muerte y que no se toma conciencia de una realidad

\subsection{Estudio de los metales pesados}

Se denomina metales pesados a aquellos elementos químicos que poseen un peso atómico comprendido entre $63,55(\mathrm{Cu})$ y $200,59(\mathrm{Hg})$, y que presentan un peso especifico superior a 4 $\left(\mathrm{g} / \mathrm{cm}^{3}\right)$. Destacar que en ésta categoría entran prácticamente todos los elementos metálicos de interés económico.

Asimismo, es de destacar que hasta el presente, no se dispone de una definición oficial generalmente aceptada, ni de un listado de esos elementos, ni de una referencia clara y exacta de las propiedades o caracteres de los metales pesados que provenga de alguna sociedad científica u organismo referente de alto nivel, como podría ser la IUPAC (Unión Internacional de Química Pura y Aplicada) o la US EPA. [2]. Los metales pesados se identifican por sus propiedades tales como: Cadmio (Cd), Cobre $(\mathrm{Cu})$, Plomo $(\mathrm{Pb})$, Cromo $(\mathrm{Cr})$, Mercurio $(\mathrm{Hg})$, Níquel (Ni), Selenio (Se).

\subsection{Contaminación de los metales}

a) Plomo: El polvo es un metal pesado que forma de partículas de polvo, no se degrada y al ser emitido al aire puede permanecer en el medio ambiente 10 días. La concentración máxima permitida de este metal es de $0,1 \mathrm{mg} / \mathrm{l}$, ingresa al organismo por vía respiratoria, por ingestión, por la piel, provocando la enfermedad conocida como saturnismo, la cual tiene un desarrollo lento y tarda más de diez años en declararse abiertamente. Él anormal intercambio de oxígeno y dióxido de carbono, pasa a la sangre provocando anemia, y afecta los diferentes órganos del cuerpo humano.

El polvo de plomo provoca alteraciones al sistema nervioso, produciendo alteraciones de carácter, irritabilidad, insomnio, dificultad en la concentración y hasta disminución del lívido. Cuando el metal se acumula en los huesos impide la producción de sangre. Cuando la intoxicación está avanzada, provoca dolores estomacales agudos, línea azul en las encías, caída de la muñeca, temblores y convulsiones, problemas renales, terminando así con la vida por insuficiencia renal. El polvo de $\mathrm{Pb}$. provoca alteraciones al sistema nervioso.

b) Mercurio: El mercurio ingresa al cuerpo por inhalación de vapores de mercurio, por ingestión de metilmercurio por el consumo de agua, alimentos contaminados: y por penetración en la piel aunque menos frecuentemente provoca una acción irritante y a veces cáustica sobre la piel y la mucosa.

Aproximadamente el $80 \%$ es absorbido por los pulmones, una importante cantidad de mercurio se concentra en el riñón, cerebro, hígado, glóbulos rojos de la sangre. Es eliminado fundamentalmente por la orina, heces, sudor, saliva, encontrándose también en la leche materna. La concentración ambiental máxima permitida de mercurio por la OMS es de $0,04 \mathrm{mg} / \mathrm{m}^{3}$.

En la piel: Irritaciones cutáneas, Sistema respiratorio, Dolor en el pecho dificultad para respirar, tos, etc. 


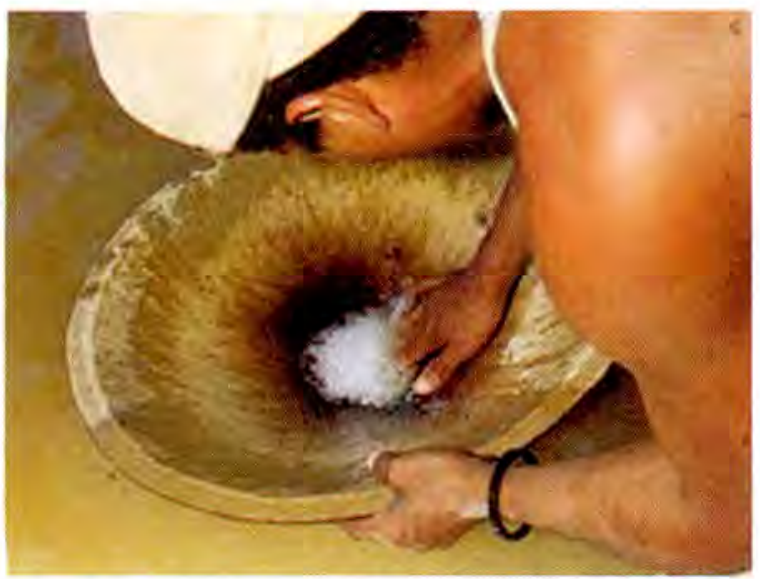

Figura $\mathbf{N}^{0}$ 1: Contacto del Mercurio con la piel.

Sistema nervioso: Sabor metálico en la boca, náusea, diarrea, dolor de cabeza, dolores musculares, alteraciones visuales y auditivas y mentales. Inflamación de las encías, inflamación de los riñones, temblor de músculos. La muerte sobreviene por edema, destrucción del tejido pulmonar, insuficiencia renal. Cuando la intoxicación es crónica, se produce los siguientes síntomas: Temblores con movimientos toscos, y sacudidas que comienzan en los dedos de las manos, párpados, labios y lengua, luego brazos, piernas y cabeza.

c) Arsénico: En las fundiciones de plomo, zinc y cobre se encuentra el As; siendo un metal pesado que no se degrada, no se evapora. Los compuestos que forman este metal son solubles en agua. En la actualidad se conocen que el arsénico y sus compuestos son cancerígenos. Penetra al organismo por medio de la piel causando irritación a la misma. Por inhalación, los vapores entran a los pulmones, irritando las vías respiratorias.

La contaminación de arsénico en la piel es el aparecimiento de manchas claras y obscuras, formando pequeños "callos" en las palmas de las manos, plantas de los pies que pueden transformarse en cáncer de la piel. El arsénico produce además daño genético como malformaciones oculares y renales; aborto y complicaciones en el embarazo.[1]

d) Cadmio: Los minerales de cadmio, no se encuentran en concentraciones y cantidades suficientes como para justificar una actividad minera específica por el elemento. Entre los minerales de cadmio, la greenockita (CdS) es el más común. Este mineral se encuentra casi siempre asociado con la esfalerita ( $\mathrm{ZnS})$. De esta manera, el cadmio se recupera principalmente como un subproducto de la minería, fundición, y refinación del zinc. Debido a su toxicidad, el cadmio se encuentra sujeto a una de las legislaciones más severas en términos ambientales y de salud humana.

f) Asbesto: El asbesto es una fibra, compuesta principalmente por sílice y oxígeno, además de calcio, magnesio, hierro y sodio. Sus efectos pueden dividirse en no malignos y malignos. Entre las formas no malignas, la más notable es una neumoconiosis fibrogénica denominada asbestosis, que parece ser irreversible aún detenida la exposición y entre las formas malignas, un tipo de cáncer propio de las serosas pleurales y peritoneales, denominado mesotelioma. Se trata de un cáncer de rápida evolución y alta mortalidad asociado al asbesto, a las distintas variedades de fibra.

\section{RESULTADOS}

\subsection{Evaluación del Plomo}

El plomo se encuentra presente en un gran número de minerales, siendo la forma más común el sulfuro de plomo (galena: PbS). También son comunes, aunque en orden decreciente, la cerusita $\left(\mathrm{PbCO}_{3}\right)$ y la anglesita $\left(\mathrm{PbSO}_{4}\right)$. El plomo es un metal difícilmente movilizable. El particuldo fino de $\mathrm{Pb}$ $(10-100 \mu \mathrm{m})$ puede ser peligroso por las siguientes razones:

- Se adhiere más fuertemente a la piel.

- Es más soluble que el particulado grueso en el tracto gastrointestinal.

- Es fácilmente absorbible a través del sistema respiratorio.

En adultos que trabajan en ambientes expuestos a la contaminación con plomo, el metal puede acumularse en los huesos, donde su vida media es superior a los 20 años. La osteoporosis, embarazo, o enfermedades crónicas pueden hacer que éste plomo se incorpore más rápidamente a la sangre.

- Respiratorio: En estudios experimentales hay unos incrementos de neumonías asociados a niveles elevados de plomo en aire. Se ha detectado inhibición de fagocitos, y distintos efectos sobre la inmunidad pulmonar.

- Renal: Existen reportes de correlación de plomo con daño tubular, con excreción de proteínas marcadores, evidencias de deterioro de la función renal en sujetos con cifras de plomo plasmático elevado.

- Neurológico: A niveles de bajas dosis existen evidencias de daño neurológico y neuro comportamental. Si bien las variaciones entre 
grupos con niveles de plomo sobre la norma, desplazan la media de CI en 4 a 7 puntos, dada la forma sigmoidea de la curva, el efecto de una mediana que se desplaza 6 puntos, es de un incremento cuatro veces de los niveles de déficit intelectual severo $(\mathrm{CI}<80)$.

- Reproducción: Estudios asocian la sostenida baja del recuento de espermios en Europa en las últimas décadas a la toxicidad de plomo. Se ha demostrado una marcada diferencia en el recuento en trabajadores expuestos a plomo. En ratas los estudios muestran daño epididimal y testicular.

- Carcinogénesis: existen asociaciones en trabajadores expuestos a plomo, con mayor mortalidad por tumores en general. La Agencia internacional para Investigación del Cáncer lo clasifica como $2 \mathrm{~b}$, es decir con evidencia adecuada en animales de carcinogenicidad, pero no en humanos.

\subsection{Evaluación del Mercurio}

La forma principal de mercurio en la naturaleza es el cinabrio ( $\mathrm{HgS})$, el que constituye la mena principal, al ponerse en contacto con un ambiente acuático, el mercurio se transforma en metilmercurio, un potente neurotóxico. Las principales fuentes de contaminación por mercurio son las naturales debido a los desprendimientos o el desgaste de la corteza terrestre, y la causada por el hombre en los procesos industriales. Nuevas investigaciones sugieren que el mercurio puede viajar largas distancias en la atmósfera, combinándose con otros agentes químicos cuando viaja, y son mucho más solubles en agua.

\subsection{Evaluación del Arsénico}

Los compuestos más usados son:

- Ácido Arsénico $\left(\mathrm{H}_{3} \mathrm{AsO}_{4}\right)$; Contiene un $50 \%$ de arsénico y se utiliza en la producción de arseniatos.

- Tetracloruro de Arsénico $\left(\mathrm{AsCl}_{3}\right)$; Contiene un $76 \%$ de Arsénico y su utiliza en la industria de la cerámica y la fabricación de que contienen cloro.

- Trióxido de Arsénico (As2 O3); También contiene un $76 \%$ de As y se utiliza como materia prima de compuestos de Arsénico, en la fabricación de vidrio, en taxidermia y como compuesto de insecticidas y rodenticidas.

-Arseniato de Calcio $\left(\mathrm{Ca}_{3}\left(\mathrm{AsO}_{4}\right)_{2}\right)$ Contiene un $38 \%$ de arsénico. Se utiliza como Insecticida y molusquicida.

\subsection{Evaluación del Cadmio}

El Cd se puede adquirir por dos vías: ingestión e inhalación. Sus efectos pueden ser divididos en dos categorías:

- Agudos: fiebre de vapores de metal (metal fume fever) causada por una exposición severa; los síntomas son equivalentes a los de la gripe; en 24 horas se desarrolla generalmente un edema pulmonar agudo, el que alcanza su máximo en 3 días; si no sobreviene la muerte por asfixia, el problema puede resolverse en una semana.

- Crónicos: la consecuencia más seria del envenenamiento por cadmio es el cáncer. Los efectos crónicos que primero se observan son daño en los riñones. Se piensa que el cadmio es también el causante de enfisemas pulmonares y enfermedades de los huesos (osteomalacia y osteoporosis). Otros problemas incluyen anemia, decoloración de los dientes, y pérdida del sentido del olfato (anosmia).

$\mathrm{Al}$ explosionar $\mathrm{Cd}$ por períodos cortos produce irritación de las vías respiratorias, toxicidad pulmonar y bronquitis. La concentración permitida es de $0,005 \mathrm{mg}$. Su principal vía de acceso es la digestiva, debido al consumo de alimentos y agua contaminada. Otra vía, es la respiratoria por inhalación de aire contaminado.

\subsection{Evaluación del Asbesto}

El asbesto es una fibra, compuesta principalmente por sílice y oxígeno, además de calcio, magnesio, hierro y sodio. Sus efectos pueden ser no malignos y malignos.

Entre las formas no malignas, la más notable es una neumoconiosis fibrogénica denominada asbestosis, que parece ser irreversible aún detenida la exposición y entre las formas malignas, un tipo de cáncer propio de las serosas pleurales y peritoneales, denominado mesotelioma. Se trata de un cáncer de rápida evolución y alta mortalidad (meses a un año) asociado al asbesto, a las distintas fibras [4].

\section{IV.DISCUSIÓN}

\section{Evaluación clínico-médico}

$\mathrm{Al}$ efectuar la evaluación clínico-médico en las personas que han sido contaminados por los metales pesados, se observa los diferentes daños que ocasiona en sus órganos, debiendo seguir un tratamiento riguroso en un tiempo prolongado. 
El Plomo ante una exposición crónica afecta: Médula ósea, Gónadas, Riñón.

Debe realizarse exámenes clínicos con orientación neurológica y cardiovascular. Además hemograma, orina: beta-2, Uricemia

Microglobulina, urea sanguínea, creatinina plasmática.

Plombemia, parámetro de exposición, debiendo de realizarse la toma de la muestra de sangre (anticoagulada) se puede hacer en cualquier momento (no crítica), refrigerada a una temperatura de $4^{\circ} \mathrm{C}$ se mantiene por tres semanas, y se conserva mayor tiempo a temperaturas -18 a $20^{\circ} \mathrm{C}$.

Valor normal: < a $30 \mathrm{mcg} / 100 \mathrm{ml}$ de sangre. Actualmente se ha fijado el indice biológico de exposición en $30 \mathrm{mcg} / 100 \mathrm{ml}$ de sangre.

Plombemia que varia en distintos grados: < a 30 $\mathrm{mcg} / 100 \mathrm{ml}$. a $>80 \mu \mathrm{g} / 100 \mathrm{ml}$. con las siguientes manifestaciones de enfermedad: Anemia, compromiso renal, encefalopatía y neurológico.

El Mercurio ante una exposición en la Industria, se produce la intoxicación aguda. En altas concentraciones de vapor puede ocasionar neumonitis química y edema agudo de pulmón. Se tiene que realizar exámenes clínicos con orientación Neurológica, Psiquiátrica, Dermatológica. Gastroenterológica, concluyendo con un análisis de orina y de sangre.

Cuando el Indice Biológico de Exposición: 15 $\mathrm{mcg} / \mathrm{l}$. las consecuencias son funestas como: Timidez excesiva, pérdida del autocontrol, cambios de humor, pérdida de la memoria.

El Arsénico ante una exposición crónica se debe de hacer una evaluación clínico-médico: Análisis de orina de $100 \mathrm{ml}$., así como de sangre en $5 \mathrm{ml}$.

Dermatológica: la despigmentación en gotas (raindrop hipopigmentation) parece ser la primera manifestación cutánea de exposición crónica al As, dermatitis de contacto, melanodermia, disqueratosis palmo-plantar, disqueratosis lenticular en disco (enfermedad de Bowen), lesiones precancerosas. As en orina $>$ a $35 \mathrm{mcg} / \mathrm{g}$. de creatinina, acompañado por modificaciones transitorias en algunos de los siguientes exámenes complementarios, además de las manifestaciones clínicas: Hemograma y recuento de plaquetas, etc.

El Cadmio, presenta dos clases de toxicidad: Intoxicación aguda, al inhalar partículas de 0,002 $\mathrm{mg} / \mathrm{m}^{3}$ por humos de óxido de Cadmio. Aparece un cuadro seudogripal seguido de tos, disnea.

Exposición crónica: Este tipo de exposición se manifiesta por afectación: respiratoria, renal y trastornos óseos. Se lo considera un cancerígeno (próstata y pulmón).

Cadmio urinario varía de $>$ a $5 \mu \mathrm{g} / \mathrm{g}$. de creatinina sin proteinuria hasta $>$ a $5 \mu \mathrm{g} / \mathrm{g}$. de creatinina, antígeno prostático, la patología va en aumento, siendo necesario un control permanente en sus análisis. [7]

ElAsbesto, presenta dos casos:

\section{No maligna}

- Pulmones Asbestosis (fibrosis intersticial difusa)

- Enfermedad de las pequeñas vías aéreas (fibrosis limitada a la región peribronquial)

- Enfermedad Crónica de las vías aéreas

- Pleura Placas pleurales

-Reacciones visceroparietales (derrame pleural benigno, fibrosis pleural difusa, atelectasia redondeada).

-Piel callos de amianto.

\section{Maligna}

- Pulmones Cáncer de pulmón, Cáncer de laringe

- Mesotelioma pleural.

- Otras cavidades revestidas por mesotelio, Cáncer de estómago, esófago, colon, recto, páncreas y riñón. [7]

\section{CONCLUSIONES}

1. De todas las especies de mercurio conocidas, la más peligrosa es sin duda el metilmercurio $\left(\mathrm{CH}_{3} \mathrm{Hg}\right)$.

2. El sistema nervioso es afectado por agentes neurotóxicos: gases como el monóxido y dióxido de carbono, ácido sulfhídrico, cianuro y oxido nitroso.

3. Se denomina metales pesados a aquellos elementos químicos que poseen un peso atómico comprendido entre $63,55(\mathrm{Cu})$ y $200,59(\mathrm{Hg})$, y que presentan un peso específico superior $4 \mathrm{~g} / \mathrm{cm}^{3}$.

\section{REFERENCIAS BIBLIOGRÁFICAS}

[1] Becerril, J. y Navarrete, M.E. (1993). Recopilación, aplicación y evaluación de las tecnologías de estabilización para el tratamiento de residuos peligrosos. CENAPRED. "Coordinación de Investigación". 2da. Edic.,pgs. 46, 78-91 
[2] COVENIN (1987).- Partículas suspendidas en la atmósfera. Determinación de Plomo por espectrofotometría de absorción atómica. -.Cap. VI.

[3] IHOBE (1998): Investigación de la contaminación del suelo. Vol. 2. Departamento Medio Ambiente.pgs. 27-38

[4] López Julián, P.L. \& Mandado Collado, J.M. (2002): Extracciones químicas secuenciales de metales pesados. 2 da. Edic. 110, 117, 134

[5] Razo S.I. (2002). Evaluación de la contaminación por metales y del riesgo en salud en un sitio minero de sulfuros polimetálicos: caso de Villa de la Paz-Matehuala S.L.P(México).
[6] Roychowdhury T., Uchino T., Tokunaga H. \& Ando M . (2002): Arsenic and other heavy metals in soil from an arsenic-affected area of West Bengal, India. Chemosphere. Pags: 6: 605-618

[7] Velásquez H., Molina B., Morales J., Cano Y. (2006).- Niveles de contaminación por gases ácidos $\left(\mathrm{SO}_{2}, \mathrm{NO}_{2}\right)$ y partículas inhalables en dos sitios de la ciudad de Maracaibo, Venezuela.- 4ta. Edic., Cap.2.

\section{Correspondencia:}

Julio Vargas Paniagua Modesto Molina $\mathrm{N}^{\circ} 1010$

Ciudad Universitaria Fundo "Los Granados" Av. Miraflores s/n Tacna Perú jvpvargas@hotmail.com 